\title{
Novel ynamide structural analogues and their synthetic transformations
}

\author{
Ting Lu* and Richard P. Hsung* \\ Division of Pharmaceutical Sciences, School of Pharmacy, University of Wisconsin, Madison \\ Wisconsin 53705, United States \\ E-mail: luting80@yahoo.com, rhsung@wisc.edu
}

DOI: http://dx.doi.org/10.3998/ark.5550190.p008.512

\begin{abstract}
This Highlight accounts for a recent phenomenon in which a series of novel ynamide structural analogues have emerged and caught the attention of the synthetic community. Preparations and reactions of these de novo ynamide variants are delineated here to demonstrate their accessibility as well as their reactivity. This Highlight should help reveal that these unique $N$-containing alkynes can become highly versatile building blocks in organic syntheses.
\end{abstract}

Keywords: Ynimides, yne-hydrazides, amidinyl-ynamides, yne-sulfoximines, yne-imines, diaminoacetylenes

\section{Table of Contents}

1. Introduction: Ynamide Analogues

2. Discussions: Syntheses and Reactions

2.1 Diaminoacetylenes

2.2 Ynimides

2.3 Yne-imines

2.4 Yne-hydrazides

2.5 Amidinyl-ynamides

2.6 Yne-sulfoximines

3. Conclusions 


\section{Introduction: Ynamide Analogues}

Ynamides 1 (Scheme 1), the electron-deficient alternatives of the important but labile ynamines, ${ }^{1-14}$ represent one of the most significant and versatile $N$-containing building blocks in organic synthesis. ${ }^{15-17}$ Particularly, in the past 15 years, interests in this versatile building block have raised dramatically. ${ }^{18-21}$ This boom is closely related to the fact that rapid development of efficient synthesis methods ${ }^{22-26}$ has rendered ynamides highly accessible. Very recently, preparations of structural analogues of ynamides have emerged as novel $\mathrm{N}$-containing alkyne building blocks. ${ }^{27-36}$ These preparations mainly are based on modified methods for ynamide synthesis $^{22-26}$ as described in Scheme 1. With the belief that these novel analogues will also become versatile synthons that will be tremendously useful to organic synthesis, especially in the arena of $N$-heterocycle constructions, we highlight herein syntheses and reactions of de novo ynamide analogues: diaminoacetylenes $\mathbf{2}$, ynimides $\mathbf{3}$, yne-imines $\mathbf{4}$, yne-hydrazides $\mathbf{5}$, amidinylynamides 6, and yne-sulfoximines 7 (Scheme 1).

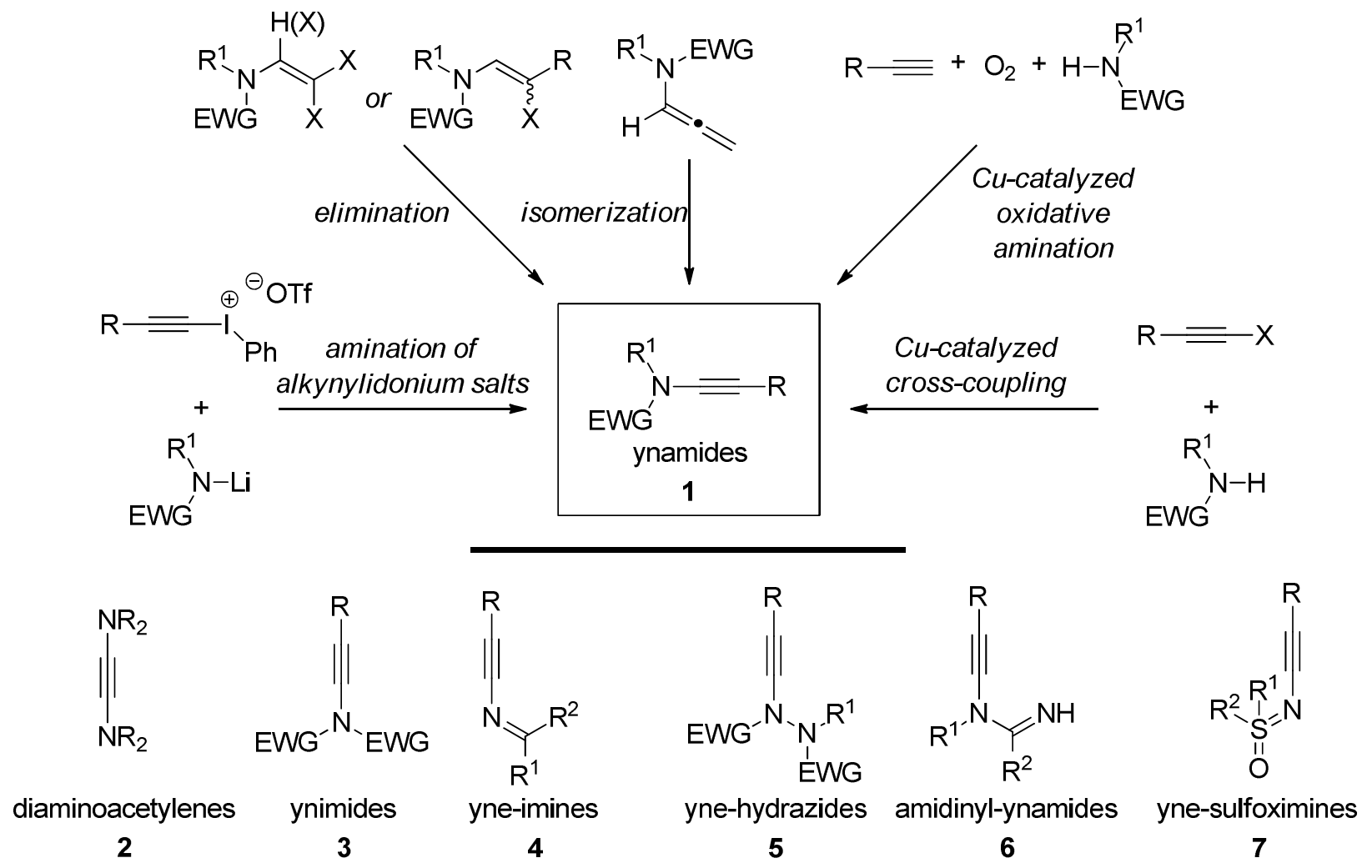

\section{Scheme 1}




\section{Discussions: Syntheses and Reactions}

\subsection{Diaminoacetylenes}

Tamm $^{27}$ described a novel approach to access diaminoacetylenes $\mathbf{1 0}$ by Fristch-ButtenbergWiechell (FBW) rearrangement ${ }^{37-39}$ of 1,1-dihalo-2,2-ethenediamines 8 via the LiBr-bound carbenoids 9 (Scheme 2). This preparation of diaminoacetylenes $\mathbf{1 0}$ led to their X-ray structures for the first time, thereby revealing a virtually perpendicular orientations for the two $\mathrm{NC}_{3}$ planes (See $P 1$ and $P 2$ in box at right in Scheme 2). ${ }^{27}$

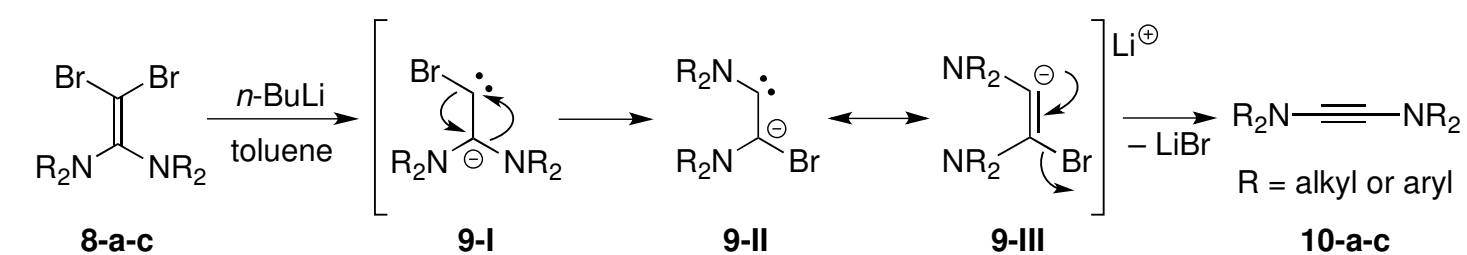

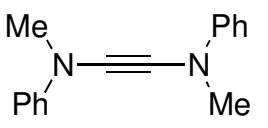

10-a: $92 \%$

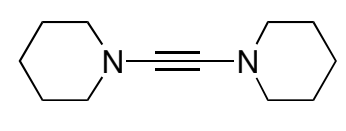

10-b: $89 \%$

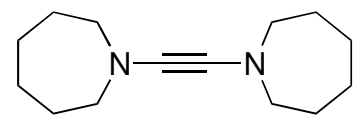

10-c: $81 \%$
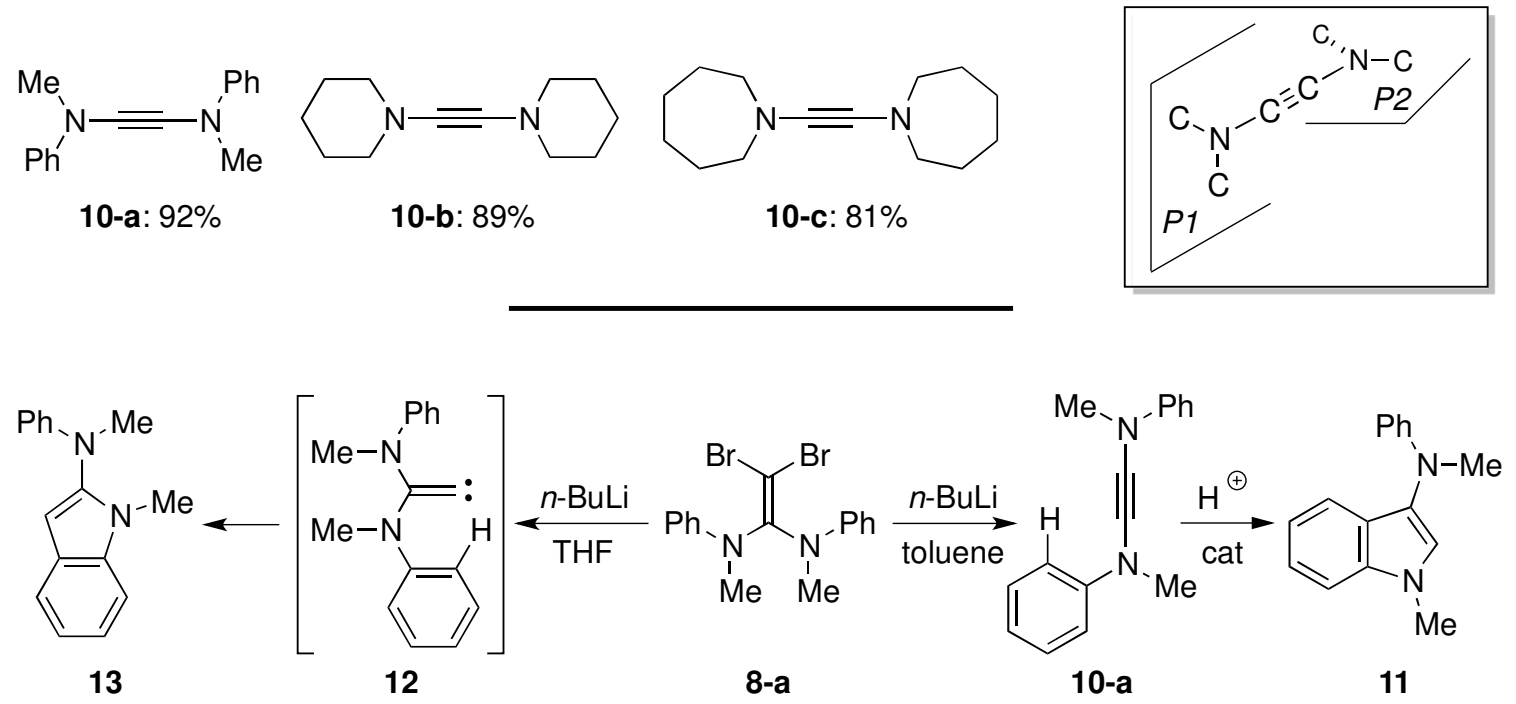

\section{Scheme 2}

With Brønsted acid or Lewis acid serving as catalysts, diaminoacetylene 10-a would undergo an intramolecular hydroarylation to afford indole 11. It is noteworthy that with polar solvent such as THF, 8-a is not subjected to FBW rearrangement. Instead, indole $\mathbf{1 3}$ was formed via an intramolecular $1,5 \mathrm{C}-\mathrm{H}$ insertion of the vinylidene intermediate $12 .{ }^{27}$

The successful synthesis of 1,2-dipiperidinoacetylene 10-b enabled studies of coordination chemistry on diaminoacetylenes through preparations of a series of structurally interesting metal complexes such as monometallic complex 14, homobimetallic complex 15, and heterobimetallic complex 16 (Scheme 3). ${ }^{40}$ 


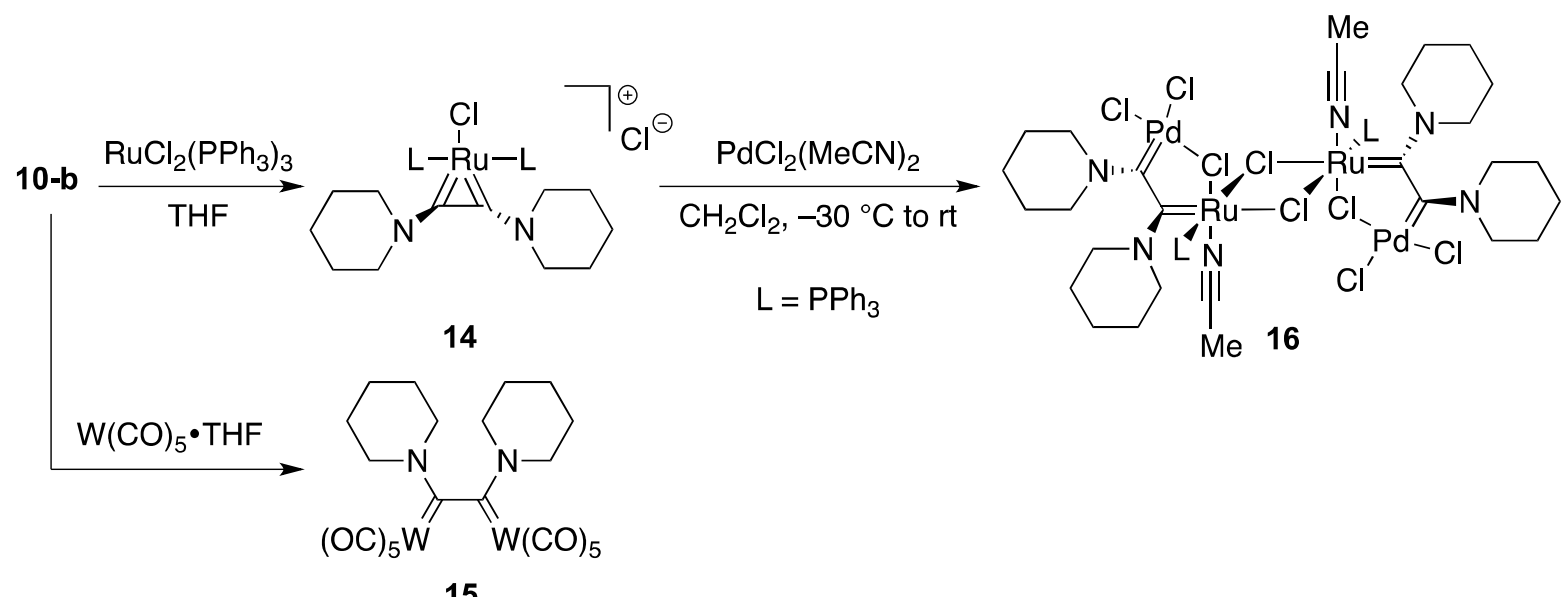

\section{Scheme 3}

\subsection{Ynimides}

Sueda $^{28}$ reported an efficient synthesis of ynimides 19 via a copper catalyzed amidation of alkynyl(trialryl)bismuthonium salts 17. An application of these de novo $N$-ethynyl phthanlimides was demonstrated through a copper catalyzed [3+2] cycloaddition followed by hydrazinolysis, leading to 4-amino-1,2,3-triazole 22. This transformation represents as an alternative preparation of 22 to the existing [3+2] cycloaddition using the highly labile ethynamine.

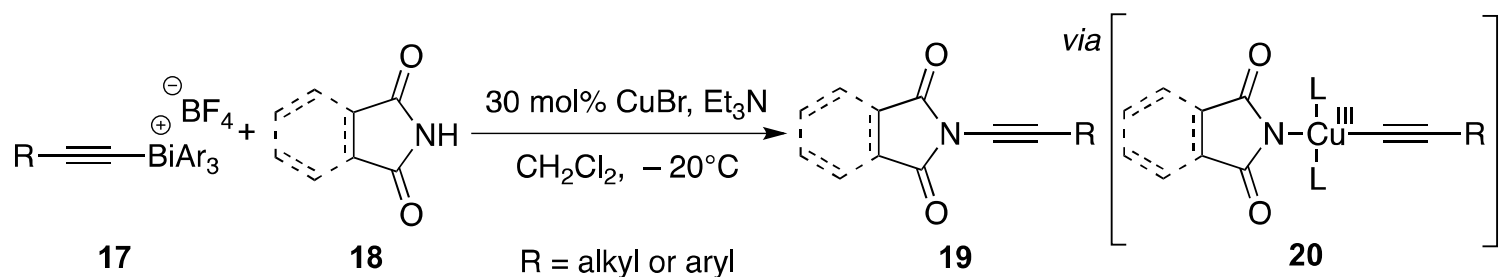

$$
\mathrm{Ar}=p-\mathrm{MeC}_{6} \mathrm{H}_{4} \text { or } p-\mathrm{MeO} \mathrm{C}_{6} \mathrm{H}_{4}
$$<smiles>CS(=O)(=O)C#CN1C(=O)c2ccccc2C1=O</smiles>

19-a: $53 \%$<smiles>O=C1c2ccccc2C(=O)N1C#CC=[V]</smiles>

19-b: $61 \%$<smiles>CC(C)(C)C#CN1C(=O)C=CC1=O</smiles>

19-c: $52 \%$<smiles>O=C1CCC(=O)N1C#Cc1ccccc1</smiles>

19-d: $51 \%$

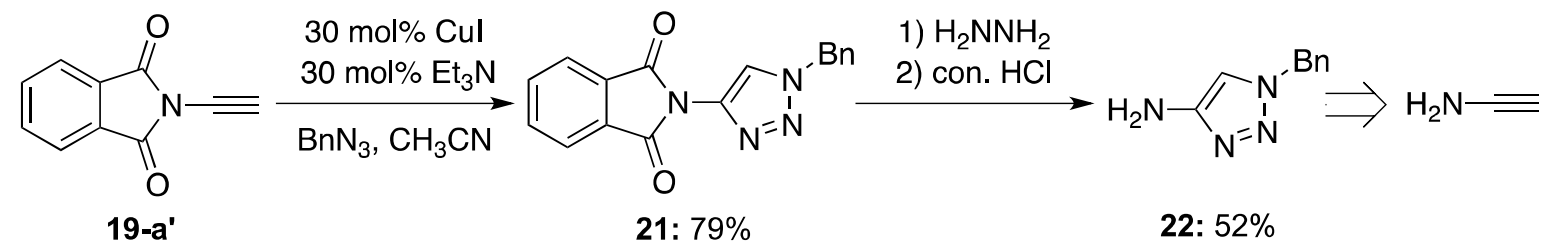

\section{Scheme 4}


In addition, with the aid of an $\mathrm{Au}(\mathrm{I})$ catalyst, which is a preferred $\pi$ electrophilic Lewis acid, ynimides 19 reacted with methanol to afford $\beta$-ketoimides 23. On the other hand, with $\operatorname{Ag}(\mathrm{I})$ catalyst, oxazoles 24 were formed through an initial coordination of $\mathrm{Ag}(\mathrm{I})$ with the carbonyl oxygen (see 27). Similarly, 19-e could react with aniline, under $\mathrm{Au}(\mathrm{I}) / \mathrm{Ag}(\mathrm{I})$ or $\mathrm{Ag}(\mathrm{I})$ only catalysis, to afford phthalimide 29 and oxazolylbenzamide 30, respectively (Scheme 5). ${ }^{29}$

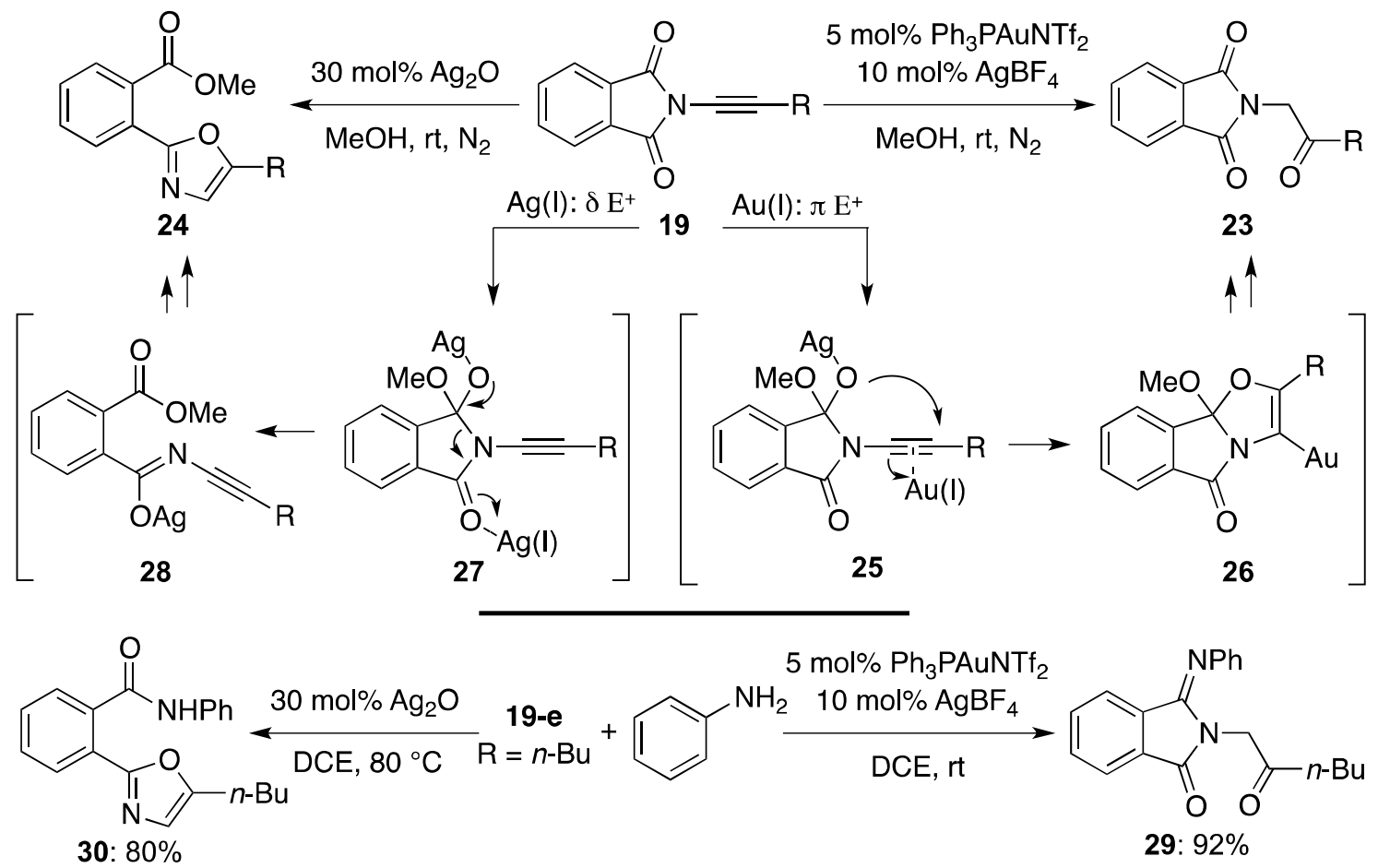

\section{Scheme 5}

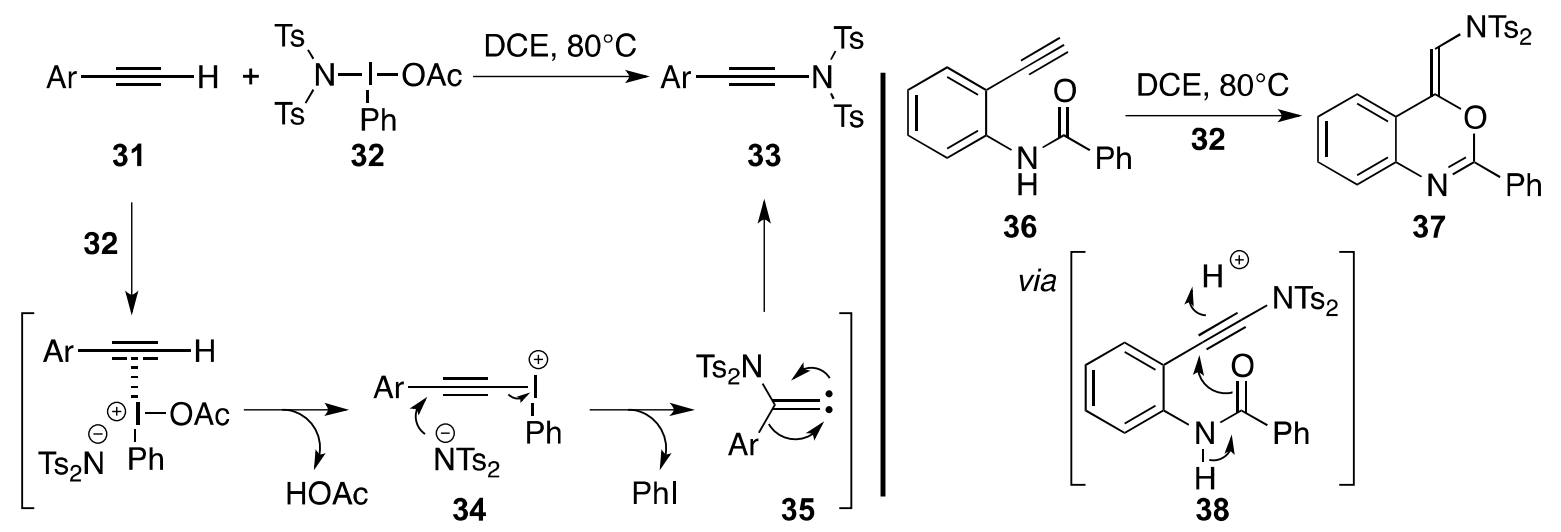

\section{Scheme 6}


Muñiz $^{30}$ later demonstrated a metal-free amination of aryl substituted terminal alkynes 31 using hypervalent iodine reagent $\mathbf{3 2}$ to synthesize ynimides $\mathbf{3 3}$. When treating the aniline derived acetylene 36 with 32, benzo-1,3-oxazine $\mathbf{3 7}$ was obtained likely through the ynimide intermediate 38 (Scheme 6).

\subsection{Yne-imines}

Evano $^{31}$ documented syntheses of yne-iminies $\mathbf{4 1}$ through copper catalyzed oxidative crosscouplings of terminal alkynes 39 with diaryl imines 40. By using Lindlar's catalyst, these de novo yne-imines 41 could be reduced to afford Z-azadienes 42. On the other hand, the addition of MeLi induced dimerization of $\mathbf{4 1}$ led to the highly substituted azines $\mathbf{4 3}$ (Scheme 7).

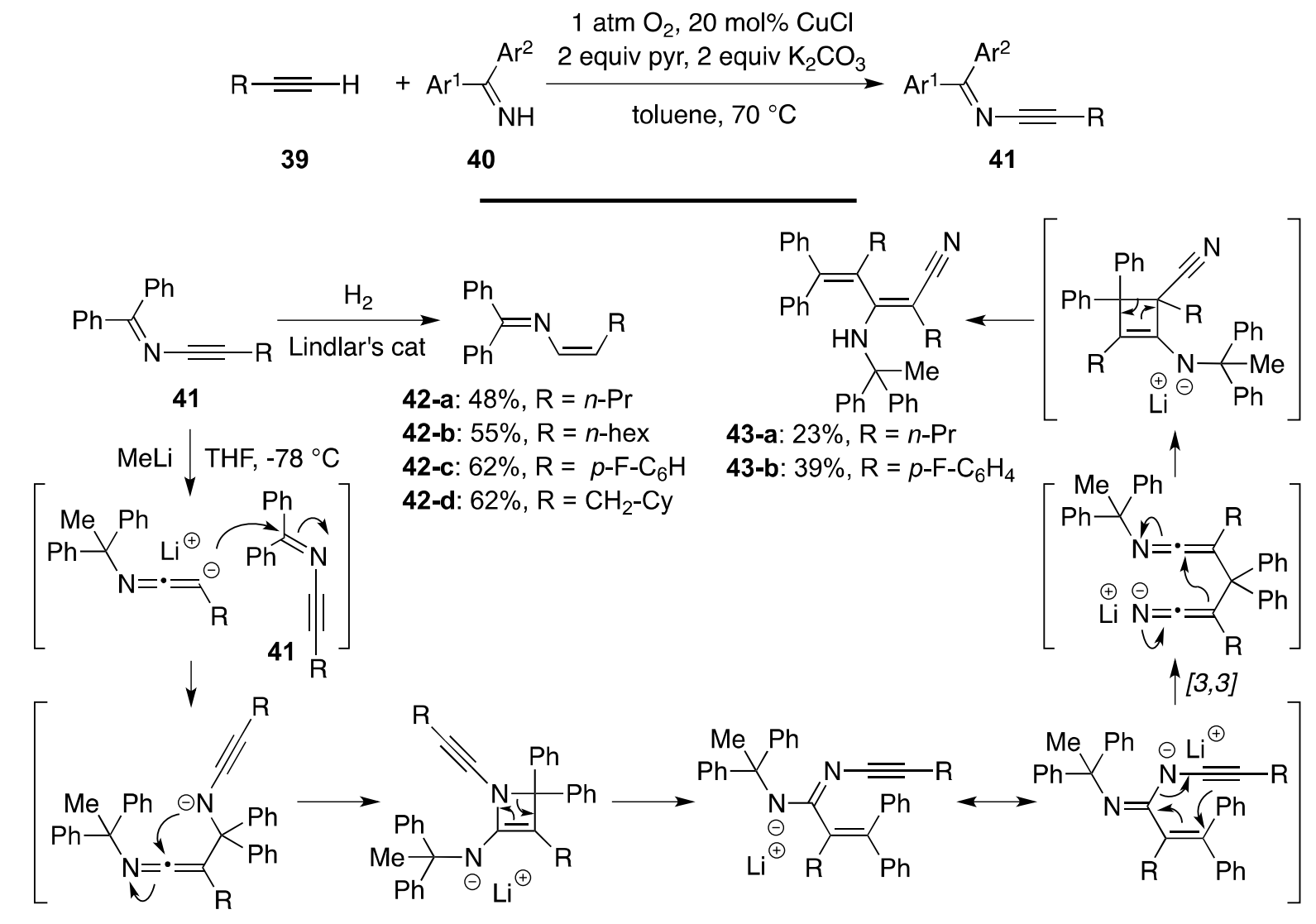

\section{Scheme 7}

\subsection{Yne-hydrazides}

Batey $^{32}$ reported a concise synthesis of yne-hydrazides $\mathbf{4 6}$ through a straightforward addition of lithiated acetylenes 44 to diazodicarboxylates 45 (Scheme 8). These novel yne-hydrazides were shown as useful building blocks in a series of interesting transformations. In addition to a Larock-type indole formation (Scheme 8), ${ }^{41}$ Batey showcased syntheses of structurally unique heterocycles such as $\mathbf{4 8}, \mathbf{5 0}$, and $\mathbf{5 2}$ through a sequence of [3+2] cycloaddition followed by 
condensation under acidic conditions (Scheme 9). It is noteworthy of the complete regioselectivity switch in the $[3+2]$ cycloaddition step between using $\mathrm{Cp} * \mathrm{RuCl}(\mathrm{COD})$ and $\mathrm{Cu}(\mathrm{OAc})_{2}$ (see 47 versus 49), ${ }^{42}$ albeit the former used an internal yne-hydrazide, while the latter employed a terminal yne-hydrazide.

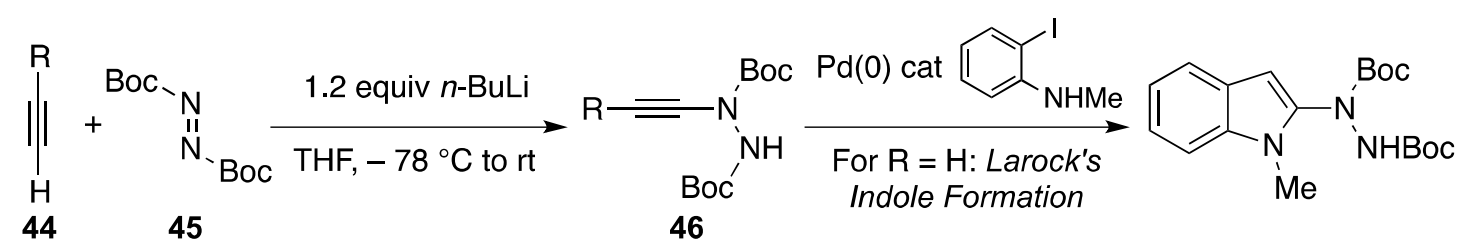

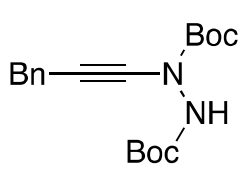

46-a: $53 \%$

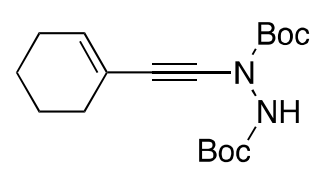

46-b: $87 \%$

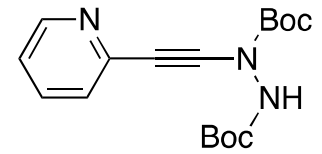

46-c: $30 \%$<smiles>CC(C)(C)OC(=O)NN(C#Cc1ccsc1)C(=O)OC(C)(C)C</smiles>

46-d: $55 \%$

\section{Scheme 8}

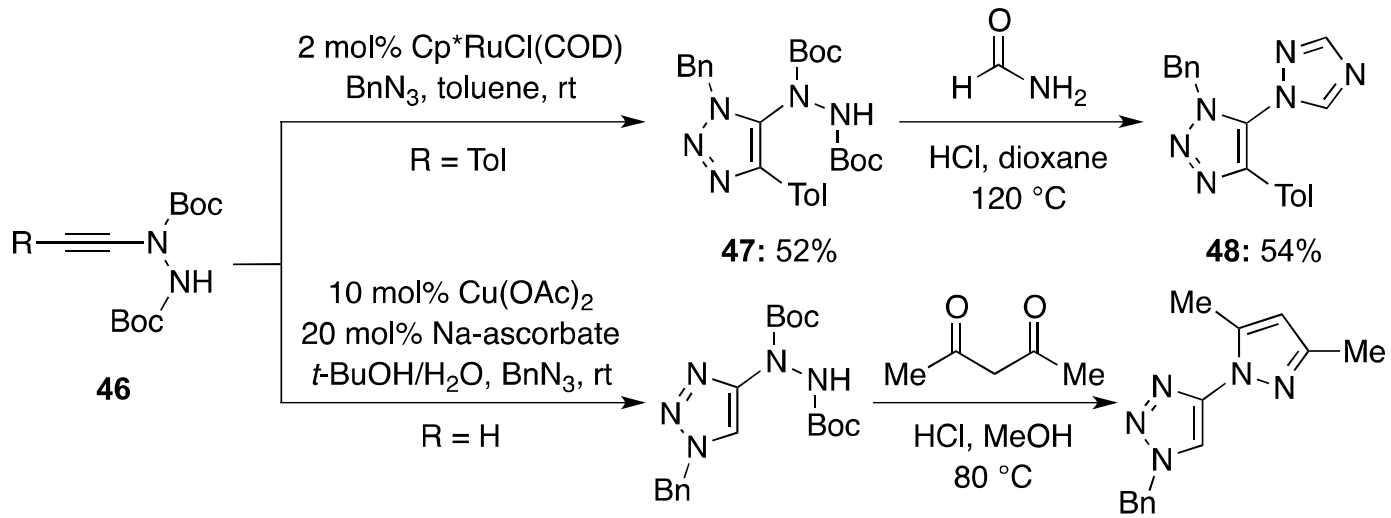

49: $68 \%$

50: $51 \%$

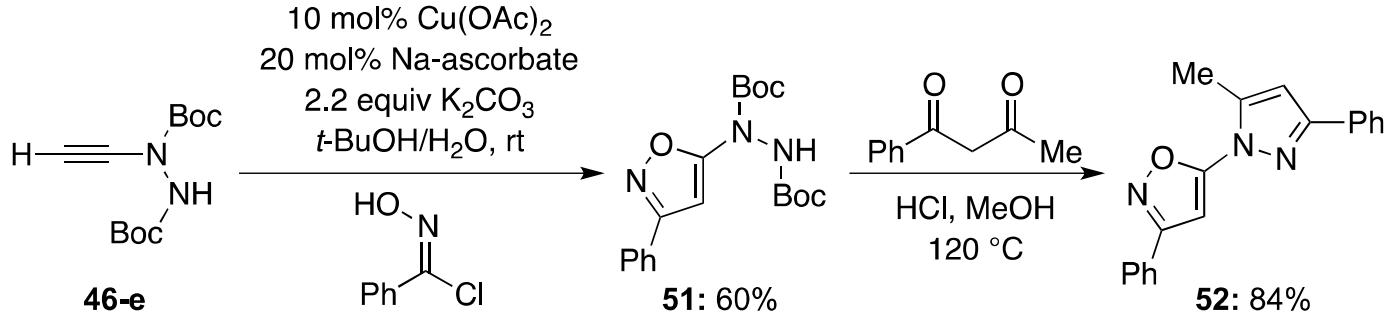

\section{Scheme 9}

Recently, Batey ${ }^{33}$ utilized yne-hydrazide 46-f to synthesize Z-ene-hydrazide 54, which 
represent an analogue of biologically active natural product hydrazidomycin $\mathrm{A}^{43}$ (Scheme 10). The key Lindlar's hydrogenation approach is similar to that of Hsung's Z-enamides syntheses from ynamides. ${ }^{44}$

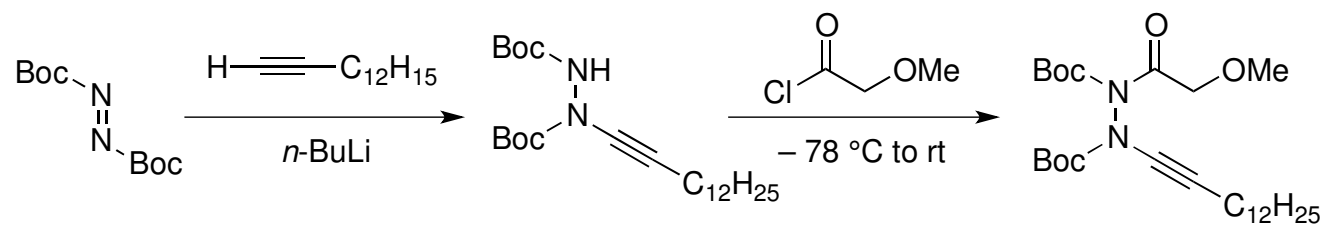

45

46-f

53: $62 \%$ from 45

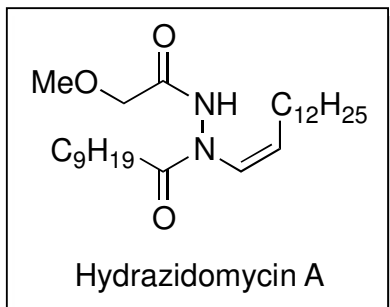

1) 10 mol\% $\mathrm{Mg}\left(\mathrm{ClO}_{4}\right)_{2}, \mathrm{MeCN}$
2) $\mathrm{H}_{2}$, Lindlar's catalyst<smiles>CCC/C=C\N(NC(=O)COC)C(=O)CCCCCCC</smiles>

54: $59 \%$ from 53

\section{Scheme 10}

\subsection{Amidinyl-ynamides}

Neuville $^{34}$ reported syntheses of 1,2,4-trisubstituted imidazoles $\mathbf{5 8}$ from terminal alkynes $\mathbf{5 5}$ and amidines 56. Amidinly-ynamides 57 are believed to be the intermediates resulting from a copper catalyzed oxidative amination of $\mathbf{5 5}$ with $\mathbf{5 6}$ (Scheme 11).

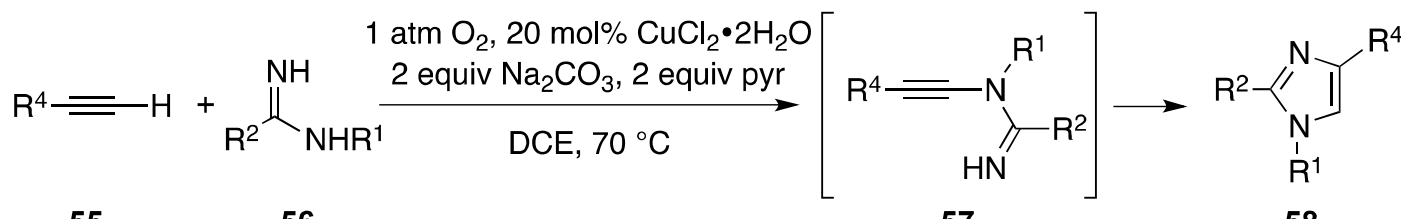

55

56

57

58

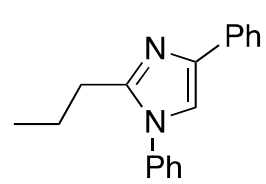

58-a: $46 \%$

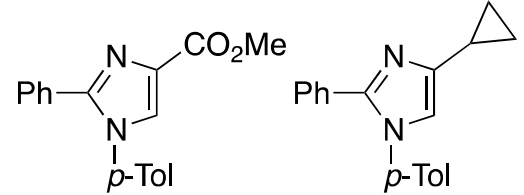

58-b: $59 \%$

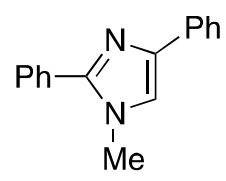

58-d: $47 \%$

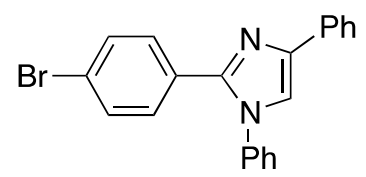

58-e: $56 \%$

\section{Scheme 11}

\subsection{Yne-sulfoximines}

Bolm $^{35}$ described the first preparations of yne-sulfoximines 61 through a copper catalyzed oxidative cross-coupling of sulfoximines 59 with terminal alkynes $\mathbf{6 0}$. These yne-sulfoximines 
61 could be transformed to $N$-acyl sulfoximines 62 via simple hydrolysis upon silica gel column chromatography (Scheme 12).

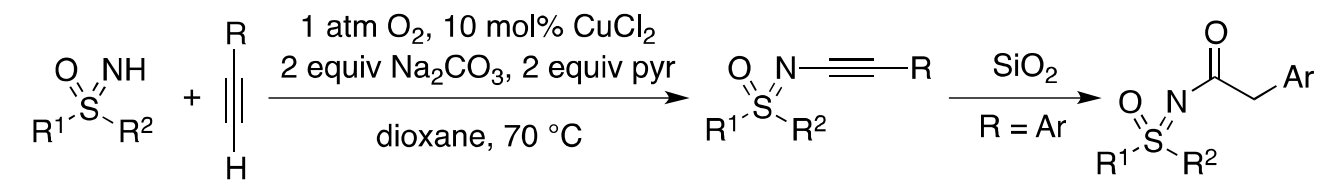

59<smiles>CS(=O)(=NC#Cc1ccc(C#N)cc1)c1ccccc1</smiles>

61-a: $78 \%$<smiles>CS(=O)(=NC(=O)Cc1ccc(C#N)cc1)c1ccccc1</smiles>

62-a: $80 \%$<smiles></smiles>

61-b: $78 \%$<smiles>CS(=O)(=NC(=O)Cc1ccccc1)c1ccccc1</smiles>

62-b: $80 \%$
61<smiles>O=S1(=NC#C[Ge]O)CCCC1</smiles>

61-c: $62 \%$<smiles></smiles>

61-d: $78 \%$

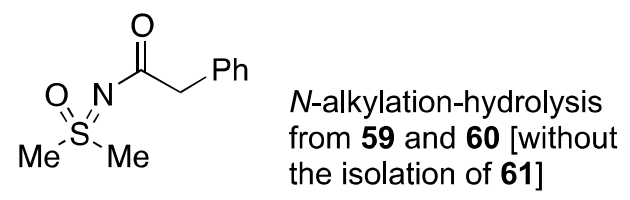

62-e: $55 \%$

\section{Scheme 12}<smiles>CC1(C)C(=O)C(c2ccccc2)=C1N=S(=O)(c1ccccc1)c1ccccc1</smiles>

63-a: $78 \%$

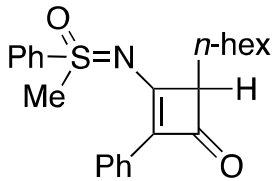

63-b: $80 \%$<smiles>[R7]C1([R7])C(=O)C([Al])=C1N=S(C)(=O)P</smiles>

63-a-d

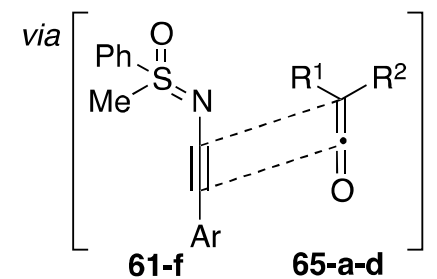<smiles>CS(=O)(=NC1=C(c2ccccc2)C(=O)C1c1ccccc1)c1ccccc1</smiles>

63-c: $81 \%$

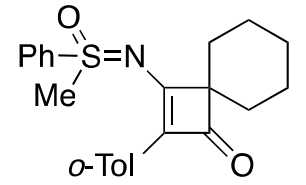

63-d: $87 \%$

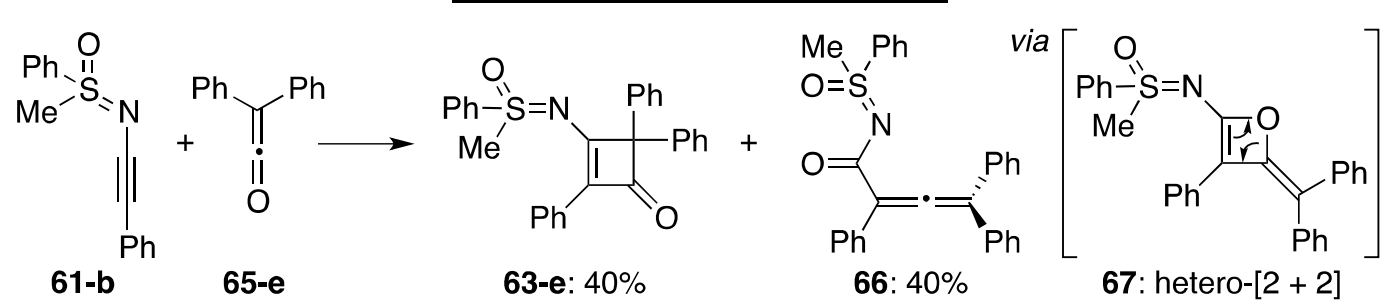

\section{Scheme 13}


Recognizing the excellent potential of yne-sulfoximines $\mathbf{6 1}$ as new synthetic building blocks, Bolm's group also explored their usage in thermal $[2+2]$ cycloaddition reactions. ${ }^{36}$ They found that yne-sulfoximines $\mathbf{6 1}$ could react with ketenes $\mathbf{6 5}$ to form the sulfoximine derived cyclobutenones 63 via a $[2+2]$ cycloaddition pathway. When using diphenyl ketene 65-e, allenyl amide $\mathbf{6 6}$ was also isolated in addition to the expected cyclobutenone 63-e. The formation of 66 is likely a result of pericyclic ring opening of the oxetene intermediate $67,{ }^{45-54}$ which could be envisioned through a hetero-[2+2] pathway (Scheme 13).

\section{Conclusions}

This Highlight presents a recent phenomenon in developing syntheses and reactions of novel structural analogues of ynamides. While their syntheses and reactivities are similar to those of ynamides in many aspects, their unique motifs have rendered them special merits in terms of reactivities. These $N$-containing alkynes should become new versatile building blocks in organic synthesis.

\section{Acknowledgements}

Authors thank NIH [GM066055] for funding.

\section{References}

1. Bode, J. Ann. 1892, 267, 268.

2. Klages, F.; Drerup, E. Ann. 1941, 547, 65.

3. Zaugg, H. E.; Swett, L. R.; Stone, G. R. J. Org. Chem. 1958, 23, 1389. http://dx.doi.org/10.1021/jo01103a617

4. Wolf, V.; Kowitz, F. Ann. 1960, 638, 33.

5. Viehe, H. G. Angew Chem. Int. Ed. 1963, 2, 477. http://dx.doi.org/10.1002/anie.196304771

6. Viehe, H. G. Angew Chem. Int. Ed. 1967, 6, 767. http://dx.doi.org/10.1002/anie.196707671

7. Viehe, H. G. Chemistry of Acetylenes. Chapter 12. Marcel Dekker: New York, 1969; pp 861-912.

8. Ficini, J. Tetrahedron 1976, 32, 448. http://dx.doi.org/10.1016/0040-4020(76)85200-3

9. Pitacco, G.; Valentin, E., in Chemistry of Functional Groups. Chapter 15, 1979; pp623-714.

10. Collard-Motte, J.; Janousek, Z. Topics in Current Chem. 1986, 130, 89. http://dx.doi.org/10.1007/3-540-15810-3_3 
11. Himbert, G. in Methoden Der Organischen Chemie (Houben-Weyl), Kropf, H.; Schaumann, E., Eds. Georg Thieme Verlag: Stuttgart, 1993; pp3267. Schaumann, E., Eds. Georg Thieme Verlag: Stuttgart, 1993, pp3267.

12. Zificsak, C. A.; Mulder, J. A.; Rameshkumar, C.; Wei, L.-L.; Hsung, R. P. Tetrahedron 2001, 57, 7575 .

\section{http://dx.doi.org/10.1016/S0040-4020(01)00681-0}

13. Mulder, J. A.; Kurtz, K. C. M.; Hsung, R. P. Synlett 2003, 1379.

14. Katritzky, A. R.; Jiang, R.; Singh, S. K. Heterocycles 2004, 63, 1455. http://dx.doi.org/10.3987/REV-04-575

15. DeKorver, K. A.; Li, H.; Lohse, A. G.; Hayashi, R.; Lu, Z.; Zhang, Y.; Hsung, R. P. Chem. Rev. 2010, 110, 5064.

http://dx.doi.org/10.1021/cr100003s

PMid:20429503 PMCid:PMC2927719

16. Evano, G.; Coste, A.; Jouvin, K. Angew. Chem., Int. Ed. 2010, 49, 2840.

http://dx.doi.org/10.1002/anie.200905817

PMid:20354981

17. Wang, X-N.; Yeom, H-S.; Fang, L-C.; He, S.; Ma, Z-X.; Kedrowski, B. L.; Hsung, R. P. Acc. Chem. Res. 2013, DOI: 10.1021/ar400193g.

http://dx.doi.org/10.1021/ar400193g

18. Ackermann, L.; Potukuchi, H. K. Org. Biomol. Chem. 2010, 8, 4503.

http://dx.doi.org/10.1039/c0ob00212g

PMid:20733972

19. Domı'nguez, G.; Perez-Castells, J. Chem. Soc. Rev. 2011, 40, 3430.

20. Weding, N.; Hapke, M. Chem. Soc. Rev. 2011, 40, 4525.

http://dx.doi.org/10.1039/c0cs00189a

PMid:21594299

21. Madelaine, C.; Valerio, V.; Maulide, N. Chem.: Asian J. 2011, 6, 2224.

http://dx.doi.org/10.1002/asia.201100108

PMid:21721132

22. Mulder, J. A.; Kurtz, K. C. M.; Hsung, R. P. Synlett 2003, 1379.

23. Dehli, J. R.;Legros, J.; Bolm, C. Chem. Commun. 2005, 43, 973.

http://dx.doi.org/10.1039/b415954c

PMid:15719090

24. Tracey, M. R.; Hsung, R. P.; Antoline, J. A.; Kurtz, K. C. M.; Shen, L.; Slafer, B. W.; Zhang, Y. In Science of Synthesis, Houben-Weyl Methods of Molecular Transformations; Weinreb, S. M., Ed.; Georg Thieme Verlag KG: Stuttgart: Germany, 2005; Chapter 21.4.

25. Evano, G.; Blanchard, N.; Mathieu Toumi, M. Chem. Rev. 2008, 108, 3054.

http://dx.doi.org/10.1021/cr8002505

PMid:18698737

26. Evano, G.; Jouvinb, K.; Coste, A. Synthesis 2013, 17. 
27. Petrov, A. R.; Daniliuc, C. G.; Jones, P. G.; Tamm, M. Chem.: Eur. J. 2010, 16, 11804. http://dx.doi.org/10.1002/chem.201002211

PMid:20839193

28. Sueda, T.; Oshima, A.; Teno, N. Org. Lett. 2011, 13, 3996.

http://dx.doi.org/10.1021/ol2014973

PMid:21710984

29. Sueda,T.; Kawada,A.; Urashi, Y.; Teno, N. Org. Lett. 2013, 15, 1560.

http://dx.doi.org/10.1021/ol400338x

PMid:23496249

30. Souto, J. A.; Becker, P.; Iglesias, A.; Muñiz, K. J. Am. Chem. Soc. 2012, 134, 15505.

http://dx.doi.org/10.1021/ja306211q

PMid:22909000

31. Laouiti, A.; Rammah, M. M.; Rammah, M. B.; Marrot, J.; Couty, F.; Evano, G. Org. Lett. 2012, $14,6$.

http://dx.doi.org/10.1021/ol2032152

PMid:22171540

32. Beveridge, R. E.; Batey, R. A. Org. Lett. 2012, 14, 540.

http://dx.doi.org/10.1021/ol2031608

PMid:22216967

33. Beveridge, R. E.; Batey, R. A. Org. Lett. 2013, 15, 3086.

http://dx.doi.org/10.1021/ol401275f

PMid:23734814

34. Li, J.; Neuville, L. Org. Lett. 2013, 15, 1752.

http://dx.doi.org/10.1021/ol400560m

PMid:23528104

35. Wang, L.; Huang, H.; Priebbenow, D. L.; Pan, F.-F.; Bolm, C. Angew. Chem., Int. Ed. 2013, 52,3478 .

http://dx.doi.org/10.1002/anie.201209975

PMid:23404753

36. Pirwerdjan, R.; Priebbenow, D. L.; Becker, P.; Lamers, P.; Bolm, C. Org. Lett. 2013, 15, 5397.

http://dx.doi.org/10.1021/o14026028

PMid:24151931

37. P. Fritsch, Justus Liebigs Ann. Chem. 1894, 279, 319.

http://dx.doi.org/10.1002/jlac.18942790310

38. W. P. Buttenberg, Justus Liebigs Ann. Chem. 1894, 279, 324.

http://dx.doi.org/10.1002/jlac.18942790311

39. H. Wiechell, Justus Liebigs Ann. Chem. 1894, 279, 337.

http://dx.doi.org/10.1002/jlac.18942790312 
40. Petrov, A. R.; Bannenberg, T.; Daniliuc, C. G.; Jones, P. G.; Tamm, M. Dalton Transactions. 2011, 40, 10503.

http://dx.doi.org/10.1039/c1dt10606f

PMid:21842057

41. Larock, R. C.; Yum, E. K. J. Am. Chem. Soc. 1991, 113, 6689.

http://dx.doi.org/10.1021/ja00017a059

42. Boren, B. C.; Naravan, S.; Rasmussen, L. K.; Zhang, L.; Zhao, H.; Lin, Z.; Jia, G.; Fokin, V. V. J. Am. Chem. Soc. 2008, 130, 8923.

http://dx.doi.org/10.1021/ja0749993

PMid:18570425

43. Ueberschaar, N.; Le Sage Tchize Ndejouong, B.; Ding, L.; Maier, A.; Fiebig, H.-H.; Hertweck, C. Bioorg. Med. Chem. Lett. 2011, 21, 5839.

http://dx.doi.org/10.1016/j.bmcl.2011.07.108

PMid:21868221

44. Zhang, X.; Zhang, Y.; Huang, J.; Hsung, R. P.; Kurtz, K. C. M.; Oppenheimer, J.; Petersen, M. E.; Sagamanova, I. K.; Shen, L.; Tracey, M. R. J. Org. Chem. 2006, 71, 4170.

http://dx.doi.org/10.1021/jo060230h

PMid:16709057

45. Shindo, M. Synthesis 2003, 2275.

http://dx.doi.org/10.1055/s-2003-42417

46. Cheng, D.; Ling, F.; Li, Z.; Yao, W.; Ma, C. Org. Lett. 2012, 14, 3146.

http://dx.doi.org/10.1021/o13012277

PMid:22671022

47. Aikawa, K.; Hioki, Y.; Shimizu, N.; Mikami, K. J. Am. Chem. Soc. 2011, 133, 20092.

http://dx.doi.org/10.1021/ja2085299

PMid:22070285

48. Saito, A.; Kasai, J.; Odaira, Y.; Fukaya, H.; Hanzawa, Y. J. Org. Chem. 2009, 74, 5644.

http://dx.doi.org/10.1021/jo900857c

PMid:19496542

49. Saito, A.; Umakoshi, M.; Yagyu, N.; Hanzawa, Y. Org. Lett. 2008, 10, 1783.

http://dx.doi.org/10.1021/ol800539a

PMid:18396889

50. You, L.; Al-Rashid, Z. F.; Figueroa, R.; Ghosh, S. K.; Li, G.; Lu, T.; Hsung, R. P. Synlett 2007, 1656.

51. Kurtz, K. C. M.; Hsung, R. P.; Zhang, Y. Organic Lett. 2006, 8, 231.

http://dx.doi.org/10.1021/o1052487s

PMid:16408882

52. Mori, S.; Shindo, M. Org. Lett. 2004, 6, 3945.

http://dx.doi.org/10.1021/o1048499f

PMid:15496070 
53. Shindo, M.; Sato, Y.; Yoshikawa, T.; Koretsune, R.; Shishido, K. J. Org. Chem. 2004, 69, 3912.

http://dx.doi.org/10.1021/jo0497813

PMid:15153025

54. Shindo, M.; Matsumoto, K.; Mori, S.; Shishido, K. J. Am. Chem. Soc. 2002, 124, 6840.

http://dx.doi.org/10.1021/ja026275r

\section{Authors' Biographies}

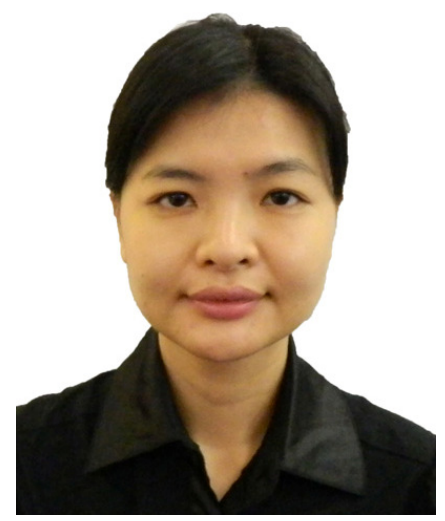

Ting Lu obtained her B.S. in Chemistry from Nankai University in China in 2002. She then went to National University of Singapore to obtain her M.S. degree in Materials Science in 2005. After that, she joined Professor Richard Hsung's research group at the University of Minnesota at Twin Cities and then the University of Wisconsin at Madison, where she studied the methodology development in cyclopropanations of enamides and allenamides, and got her Ph.D. degree in Organic Chemistry in 2009. Now she is working on new catalytic systems for the production of biorenewable chemicals from biomass at the Institute of Bioengineering and Nanotechnology, Agency for Science, Technology and Research, Singapore.

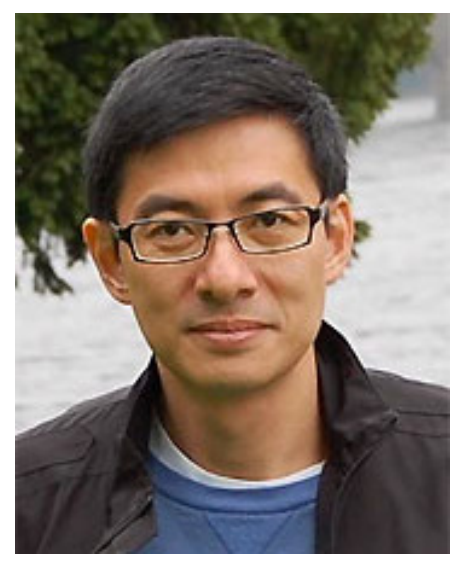

Richard P. Hsung obtained his B.S. in Chemistry and Mathematics from Calvin College in 
Grand Rapids, MI. He then attended The University of Chicago and received his M.S. and Ph.D. degrees in Organic Chemistry, respectively, under the supervision of Professors Jeff Winkler and Bill Wulff. After pursuing a postdoctoral stay with Professor Larry Sita in Chicago and NIHpostdoctoral work with Professor Gilbert Stork at Columbia University, he moved to the University of Minnesota at Twin Cities as an Assistant Professor in 1997 and was promoted to Associate Professor in 2002. He was promoted to Professor and moved to University of Wisconsin at Madison in 2006. He was a recipient of the Camille Dreyfus Teacher-Scholar Award and the National Science Foundation Career Award. He has coauthored over 200 publications, delivered over 200 invited lectures, andsupervised over 150 students and postdoctoral fellows with research interests in developing cycloaddition and annulation approaches to natural product syntheses and stereoselective methods using allenamides, ynamides, enamides, and cyclic acetals. 